The University of San Francisco

USF Scholarship: a digital repository @ Gleeson Library |

Geschke Center

$1-15-1995$

\title{
Linear Transport Theory of Magnetoconductance in Metallic Multilayers: A Real-space Approach
}

Horacio E. Camblong

University of San Francisco, camblong@usfca.edu

Follow this and additional works at: http://repository.usfca.edu/phys

Part of the Physics Commons

\section{Recommended Citation}

Camblong, Horacio E., "Linear Transport Theory of Magnetoconductance in Metallic Multilayers: A Real-space Approach" (1995).

Physics and Astronomy. Paper 11.

http://repository.usfca.edu/phys/11 


\title{
Linear transport theory of magnetoconductance in metallic multilayers: A real-space approach
}

\author{
Horacio E. Camblong \\ Department of Physics, University of San Francisco, San Francisco, California 94117
}

(Received 9 May 1994; revised manuscript received 8 August 1994)

\begin{abstract}
The linear transport behavior of metallic multilayers is studied by using the real-space Kubo formula, for a model Hamiltonian consisting of zero-range spin-dependent impurity-scattering potentials. The resulting theory allows a direct comparison with the Boltzmann equation approach in the quasiclassical limit, which is expected to be a good approximation for most magnetic multilayers. Furthermore, the regimes for which quantum corrections might be needed are explicitly indicated. It is explicitly shown that: (i) periodicity is not required as a mechanism for giant magnetoresistance, and (ii) the two main geometries, current in the plane of the layers and perpendicular to the plane of the layers, exhibit very different size effects, the latter yielding the so-called series resistor model.
\end{abstract}

\section{INTRODUCTION}

The phenomenon of negative giant magnetoresistance (GMR) has drawn considerable attention in recent years. It was first observed in $\mathrm{Fe} / \mathrm{Cr}$ superlattices, ${ }^{1}$ and later found in a large number of ferromagnetic transitionmetal/nonmagnetic-metal multilayers. ${ }^{2,3}$

Giant magnetoresistance is a magnetotransport phenomenon. In order to understand the magnetotransport problem one has to address the following two issues: how to describe transport in layered media and how to incorporate the spin degree of freedom in a transport theory. It is generally recognized that this giant effect is due to the reorientation of the magnetizations of the magnetic layers across the nonmagnetic spacers under the action of the externally applied magnetic field. The main underlying mechanism is spin-dependent scattering, first proposed in Ref. 1 , and now well established. ${ }^{4-8}$ In effect, if the conduction process is viewed as taking place in two different independent channels associated with up and down electrons (two-current $\operatorname{model}^{9}$ ), the global or measurable resistivity is the parallel combination of the resistivities of these two channels; therefore, by varying the magnetic configuration (ferromagnetic, random, partially aligned, or antiferromagnetic) one can obtain different values of the resistivity, and in particular, the configuration associated with the largest degree of order (ferromagnetic), which corresponds to the saturation field, yields a short-circuit effect between the two channels.

With these considerations in mind, two conceptually distinct transport theories have been used to account for the observed giant magnetoresistance: (i) the quasiclassical or Boltzmann-equation approach; and (ii) the quantum approach, based on the Kubo formula. ${ }^{10}$ The calculation of the ensuing transport properties poses difficulties related to the inhomogeneous nature of the scattering.

The Boltzmann-equation approach ${ }^{4,11}$ is an extension of the Fuchs-Sondheimer theory. ${ }^{12}$ It is very popular, due to its simplicity, which can be understood as a conse- quence of its treatment of inhomogeneous scattering in real space. However, it cannot be regarded as a satisfactory approach unless a justification for its use is given in terms of a quantum theory, and the conditions for its validity are correspondingly stated in this paper. Moreover, the quasiclassical approach affords an ad hoc treatment of interfaces, a problem that is resolved in this paper in a natural way by starting from its quantum counterpart.

The quantum approach ${ }^{7}$ starts from a model Hamiltonian (which accounts for impurity scattering) and uses the Kubo formula, which provides the correct quantum-statistical calculation of linear response coefficients. ${ }^{10,13,14}$ The model Hamiltonian used in Ref. 7 describes spin-dependent scattering by impurities in the different layers of the system, as well as at the interfaces; thus providing a unified description of bulk and interface scattering. Even though the approach of Ref. 7 has been regarded as experimentally successful and more fundamental than the quasiclassical approach, it has not been fully appreciated due to its apparent complexity, which is due to its treatment of inhomogeneous scattering in reciprocal space, a procedure that is reminiscent of the quantum treatment of transport in thin films by Tešanović et al. ${ }^{15}$

On the other hand, in support of the quasiclassical approach, there are some indications that the Boltzmann equation works extremely well for metallic superlattices on a phenomenological level. Moreover, as pointed out by Johnson and Camley, ${ }^{16}$ the goal of accounting for bulk and interfacial scattering in a unified way can be achieved in the quasiclassical regime by treating interfacial scattering more realistically in terms of additional thin layers representing regions of interdiffusion.

The main goal of this paper is to develop a better theoretical framework for the transition from the Kubo formula to the Boltzmann equation, a task that can be naturally accomplished by using the real-space Kubo formula. ${ }^{13,17}$ In effect, the apparent simplicity of the quasiclassical solution seems to be related to the fact that the inhomogeneous problem is tackled by solving for the distribution function in real space, where it is reduced 
to a differential equation. The real-space solution that I derive in this paper, unlike its reciprocal-space counterpart, is relatively simple and closer in form to the quasiclassical solution. The ensuing transport theory still provides a unified treatment of bulk and interface scattering, like that of Ref. 7. Furthermore, the direct parallel with the quasiclassical approach permits an exploration of the regimes for which quantum corrections are expected. Most of these results were anticipated but not derived in Ref. 8.

This paper is organized as follows. In Sec. II, I carry out a length scale analysis and define the quasiclassical regime (for which Boltzmann-equation treatments are applicable). In Sec. III, I define impurity averages as a generalization of Kohn and Luttinger's impurity averages, ${ }^{18}$ and I introduce the ensuing self-energies and diagrammatic structure. In Sec. IV, I find analytically the one-particle propagator as the solution to a differential equation in real space. In Sec. V, I develop the real-space framework of linear response in metallic multilayers, and compute the full-fledged electrical linear response, as given by the impurity-averaged two-point conductivity function. In Sec. VI, I discuss the measurable resistivities in multilayers, and I derive the peculiar current perpendicular to the plane of the layers (CPP) selfaveraging behavior. ${ }^{3,19}$ Finally, in Sec. VII, I present my conclusions and directions for future and ongoing work.

\section{LENGTH SCALE ANALYSIS AND QUASICLASSICAL REGIME}

The main goal of the transport theory of multilayered structures is to predict the so-called size effects, i.e., the dependence of transport properties with respect to the different characteristic length scales. Therefore in order to understand in more detail the physics of transport in multilayers, it is useful to analyze the length scales intrinsic to this problem and to characterize the different possible regimes.

Transport in metallic multilayers corresponds to the picture of an electron propagating throughout the whole structure and experiencing different impurity scattering rates in different regions. Therefore the first length scale is associated with the propagation of an electron through the structure. For an electron with Fermi energy $\epsilon_{F}=\hbar^{2} k_{F}^{2} /(2 m)$, and Fermi wave number $k_{F}$, the corresponding reduced Fermi wavelength $\lambda_{F}=k_{F}^{-1}$ (de Broglie wavelength at the Fermi level) is typically a "small" parameter of the order of $1 \AA$ for metallic materials (i.e., $k_{F}$ as a "large" spatial frequency). This propagation is affected by the existence of impurity scattering (dissipation), by the inhomogeneous nature of multilayers, and by the presence of outer boundaries.

First, in every layer $\mathcal{L}_{j}$ (labeled with the index $j$ ), and for each spin channel $\alpha$, there is a characteristic local mean free path $l_{j, \alpha}$ associated with the spatial damping experienced by the propagating electron. The local mean free path can be regarded as a stepwise function $l_{\alpha}(z)$ of the longitudinal coordinate $z$. It can be defined as the mean free path that the electron would have if it were propagating in an infinite structure made up of the same material and with the same type of distribution of impurities. More precisely, it is the characteristic length scale that governs the decay of the one-particle propagator in the neighborhood of the point $z$; as such, and by analogy with the case of a homogeneous metal, it can be directly extracted from the eigenvalues of the imaginary part of the self-energy $\Sigma(z)$, in the following way:

$$
l_{\alpha}(z)=\frac{\hbar^{2}}{2 m} k_{F}\left[-\operatorname{Im} \Sigma_{\alpha}(z)\right]^{-1} .
$$

Second, the inhomogeneous nature of multilayers corresponds to the fact that translational invariance is broken along the $z$ axis, as its "local" properties vary from layer to layer. Notice that, even though translational invariance is restored in the plane of the layers after impurity averaging (see Sec. III), the resulting longitudinal noninvariance is unavoidable due to layering. The corresponding inhomogeneity lengths are just the layer thicknesses $a_{j}$.

Third, the presence of the outer boundaries confines the electrons inside the metallic structure. The confinement length is the total length $L$ of the multilayered structure, and the corresponding size effects are quantum external size effects.

In this paper I show that the characteristic length parameters of multilayers make these systems behave quasiclassically. The quasiclassical regime is defined by the absence of quantum corrections: quantum size effects ${ }^{15,20}$ and quantum interference corrections, ${ }^{21}$ and of atomic inhomogeneity corrections.

Quantum size effects arise from the confinement of electrons with Fermi wave number $k_{F}$ in a finite well of size $L$. However, when $L \gg l_{j, \alpha}, \lambda_{F}$ (for all $j$ ), size effects are unaffected by the external boundaries; as these conditions are typically satisfied for the magnetic superlattices studied to date, only internal size effects will be considered in this paper (metallic sandwich structures are an exception as their total thickness is usually comparable with the mean free paths).

Quantum interference effects arise from the interference of electron paths and play a fundamental role when $\lambda_{F} \gtrsim l_{j, \alpha}$, as in the phenomenon of weak localization.

Finally, atomic inhomogeneity corrections refer to the "discreteness" of a crystal, which can only be probed on a scale of the order of the distance between atomic planes $D_{\text {at }}$, which for metallic systems is comparable to $\lambda_{F}$. On the other hand, from the viewpoint of transport properties, a length scale can be probed only with a mean free path of the same order of magnitude, because otherwise any local inhomogeneities are "averaged" over a much larger length scale. Thus the condition $l_{j, \alpha} \gg D_{\text {at }}$ guarantees that the conduction electrons propagate, with regard to their transport properties, in an "effective locally homogeneous medium." This can also be seen as an additional requirement for quasiclassical propagation.

In short, the quasiclassical regime is defined by the set of three conditions: $k_{F} L \gg 1, k_{F} l_{j, \alpha} \gg 1$, and $l_{j, \alpha} \gg D_{\text {at }}$. For metallic systems in general and for metallic multilayered structures in particular, the rela- 
tively small value of $\lambda_{F}$, which is of the same order of magnitude as $D_{\text {at }}$, makes both types of quantum corrections negligible, unless the mean free path becomes of the order of the atomic scale $D_{\text {at }}$ or $\lambda_{F}$. It should be emphasized that these conclusions refer only to transport properties, which are defined at the Fermi level, and rely upon a nearly free-electron picture and the existence of a common Fermi level for all layers, extending above local potential wells.

It should be noticed that there is another length scale in this problem, the spin-diffusion length, which is the characteristic length for spin diffusion between the two channels. For ferromagnetic metallic elements, even though electron-magnon processes become frozen at low temperatures, there exist residual spin-flip scattering processes due to spin-orbit coupling. From the experimental data of Ref. 22 and the theory of Ref. 23, one concludes that the condition of channel independence is actually fulfilled, and that in practice we can regard the spin-diffusion length as infinity.

Summarizing, if a multilayered structure is considered in the non-spin-flip limit, transport properties exhibit characteristic internal size effects governed only by (i) the inhomogeneity lengths or layer thicknesses, which characterize the spatial distribution of scatterers; and (ii) the mean free paths, which measure the strength of the scattering.

\section{IMPURITY AVERAGES AND SELF-ENERGY}

Let us now deal with the problem of impurity scattering. The impurity-dominated transport behavior at low temperatures can be described in terms of an ensemble $\mathcal{I}$ of distributions $\{\mathbf{r}\}$ of a large number $N_{\text {imp }}$ of impurities, located at positions $\mathbf{r}_{a}$, with $a=1, \ldots N_{\text {imp }}$ : $\{\mathbf{r}\}=\left\{\mathbf{r}_{1}, \mathbf{r}_{2}, \ldots, \mathbf{r}_{N_{\text {imp }}}\right\}$. For every given distribution of impurities $\{\mathbf{r}\}$, the corresponding one-electron model Hamiltonian is

$$
H=H_{0}+\sum_{a} v_{a}(\mathbf{r})
$$

where $H_{0}$ is the unperturbed free-electron Hamiltonian and $v_{a}(\mathbf{r})$ is the spin-dependent scattering potential due to an impurity or defect located at position $\mathbf{r}_{a}$. For an impurity located at the point $\mathbf{r}_{a}$, the corresponding onesite impurity potential is

$$
v_{a}(\mathbf{r})=\left(w_{a}+j_{a} \hat{\mathbf{M}}_{a} \cdot \hat{\boldsymbol{\sigma}}\right) \delta\left(\mathbf{r}-\mathbf{r}_{a}\right)
$$

which has been chosen as a zero-range potential due to the short range of the actual scattering. In Eq. (3.2) the coupling strength provides the required spin dependence through the Pauli spin vector operator $\hat{\sigma}$, which couples to the unit vector $\hat{\mathbf{M}}_{a}$ in the direction of magnetization of the respective magnetic layer. In Eq. (3.2), $w_{a}$ and $j_{a}$ are constants that measure the strength of the spin-independent and of the spin-dependent parts of the coupling strength; in particular, $j_{a} \neq 0$ for the magnetic layers and $j_{a}=0$ for the nonmagnetic layers.
A digression is now in order. An important aspect of giant magnetoresistance and transport in multilayers is that disorder at or near the interfaces seems to play an important role in the observed electrical resistances. ${ }^{1}$ The roughness of the interfaces might play some role in this, but the phenomenon of interdiffusion alone causes a significant increase in the amount of disorder (compared to the bulk value). The simplest possible model to account for this interdiffusion is therefore its representation in terms of additional "regions of interdiffusion," most simply in terms of additional thin layers, typically with a thickness of the order of $4 \AA$; this is the procedure introduced in Refs. 16 and 8. The relevance of this model lies in the fact that it not only provides a realistic picture of interface scattering but also it allows a simple treatment of the quantum theory. In Sec. IV, I indicate how this is actually implemented.

Transport properties are described in terms of "impurity-averaged" functions, such as the averaged oneparticle propagator, $T$ matrix, and two-point conductivity. The "impurity average" eliminates the dependence of transport properties with respect to specific configurations of impurities within each layer. Moreover, with the aid of the quasiclassical condition $l_{j, \alpha} \gg D_{\mathrm{at}}$, it is also defined so as to eliminate the discreteness associated with atomic sites and planes.

Let $\mathcal{R}$ be the range of the impurity potentials, $D_{\text {imp }}$ the mean interimpurity distance, and $D_{\text {inh }}$ the inhomogeneity length scale (typical layer thickness $a_{j}$ ), respectively. An arbitrary $N$-layered system consists of layers $\mathcal{L}_{j}$, with $j=1, \ldots, N$. For layer $\mathcal{L}_{j}$, let $N_{j}$ be the number of impurities.

The system is assumed to satisfy the following conditions: (i) quasiclassical regime: $L, l_{j, \alpha} \gg \lambda_{F}, D_{\text {at }}$; (ii) local homogeneity: $D_{\text {inh }} \gg D_{\text {at }}, \mathcal{R}$; (iii) locally homogeneous randomness: the impurities are distributed randomly, with a probability distribution that is uniform and statistically meaningful (that is, $N_{j} \gg 1$ ) in each layer; and (iv) dilute limit: $D_{\text {imp }} \gg \mathcal{R}, \lambda_{F}$.

The condition of local homogeneity can be trivially satisfied by having layers that consist of, at least, just a few atomic monolayers.

The property of locally homogeneous randomness yields, for an impurity in layer $\mathcal{L}_{j}$, a probability density function $\varrho_{j}(\mathbf{r})=\vartheta\left(\mathbf{r} ; \mathcal{L}_{j}\right) / V_{j}$, where $V_{j}=A a_{j}$ is the volume of layer $\mathcal{L}_{j}$, assumed to have a cross-sectional area $A$ and length $a_{j}$, and $\vartheta\left(\mathbf{r} ; \mathcal{L}_{j}\right)$ is zero everywhere except in layer $\mathcal{L}_{j}$, where it takes the constant value one. The average is performed locally and simultaneously over the whole system; thus this amounts to the following averaging procedure [see example (3.4) below and Ref. 29]:

$$
\begin{aligned}
\langle F[\{\mathbf{r}\}]\rangle_{\mathcal{I}}= & \int d^{3} r_{1} \cdots \int d^{3} r_{n} n_{\mathrm{imp}}\left(\mathbf{r}_{1}\right) \cdots \\
& \times n_{\mathrm{imp}}\left(\mathbf{r}_{n}\right) f_{\mathbf{r}_{1} \cdots \mathbf{r}_{n}}
\end{aligned}
$$

for any function $F[\{\mathbf{r}\}]=\sum_{a_{1}, \ldots, a_{n}}^{\prime} f_{\mathbf{r}_{a_{1}} \cdots \mathbf{r}_{a_{n}}}$ of the positions $\{\mathbf{r}\}$, where the sum $\Sigma^{\prime}$ excludes repeated indices; if repeated indices were included in the sum, the average should be calculated as a sum of terms, each one of which 
being similar to Eq. (3.3) but having as many integration variables and concentration factors $n_{\text {imp }}$ as distinct impurities there are. ${ }^{18,10,13,14}$ In Eq. (3.3), the local impurity concentration (volume density of impurities at position $z)$ is

$$
n_{\mathrm{imp}}(z)=\left\langle\sum_{a} \delta\left(\mathbf{r}-\mathbf{r}_{a}\right)\right\rangle_{\mathcal{I}}=\sum_{j} n_{j} \vartheta\left(\mathbf{r} ; \mathcal{L}_{j}\right)
$$

namely, a piecewise-constant or locally homogeneous concentration, with a "local" value $n_{j}=N_{j} / V_{j}$ in each layer.

The main difficulty encountered in the computation of the quantum-statistical Kubo formula for magnetic superlattices lies in their inhomogeneous nature. Let us now carry out a diagrammatic analysis with the definition of impurity-averaged quantities just introduced. In the following analysis I will omit the subscript that labels the two spin channels; actually, it will be explicitly restored in the following section.

Starting from the total potential acting on the electron,

$$
\mathcal{V}=\sum_{a} v_{a}
$$

and from the unperturbed retarded one-particle propagator $G_{0}(\varepsilon)=\left(\varepsilon-H_{0}-i 0^{+}\right)^{-1}$, one finds that the dressed propagator $G(\varepsilon)$ and the total off-shell $T$ matrix $T(\varepsilon)$ are the solutions to the integral equations

$$
G(\varepsilon)=G_{0}(\varepsilon)+G_{0}(\varepsilon) \mathcal{V} G(\varepsilon)
$$

and

$$
T(\varepsilon)=\mathcal{V}+\mathcal{V} G_{0}(\varepsilon) T(\varepsilon)
$$

respectively.

A similar scheme arises when one applies the impurityaveraging procedure described above to the infinite series that results by iteration of the integral equations (3.6) and (3.7). The only change is that the "new" irreducible insertion becomes the irreducible self-energy $\Sigma(\varepsilon)$ rather than the total potential $\mathcal{V}$. Correspondingly, the oneparticle propagator and the $T$ matrix have impurity averages given by the solution to the integral equations

$$
\langle G(\varepsilon)\rangle_{\mathcal{I}}=G_{0}(\varepsilon)+G_{0}(\varepsilon) \Sigma(\varepsilon)\langle G(\varepsilon)\rangle_{\mathcal{I}}
$$

and

$$
\langle T(\varepsilon)\rangle_{\mathcal{I}}=\Sigma(\varepsilon)+\Sigma(\varepsilon) G^{0}(\varepsilon)\langle T(\varepsilon)\rangle_{\mathcal{I}},
$$

respectively. In particular, the one-particle propagator becomes

$$
\langle G(\varepsilon)\rangle_{\mathcal{I}}=\left[\varepsilon-H_{0}-\Sigma(\varepsilon)\right]^{-1} .
$$

From the structure above, the one-particle irreducible self-energy $\Sigma(\varepsilon)$ for the impurity-averaged functions can be calculated by isolating, either in real space or in reciprocal space, the one-particle irreducible parts of the diagrammatic expansion for the impurity-averaged total (reducible) off-shell $T$ matrix $\langle T(\varepsilon)\rangle_{\tau}$. This is a straightforward generalization of the impurity averages $18,10,13,14$ for homogeneous systems; in the dilute limit the irreducible self-energy is restricted to be given essentially by the same sum of diagrams that defines the one-site offshell $T$ matrix

$$
t_{j}(\varepsilon)=v_{j}+v_{j} G_{0}(\varepsilon) t_{j}(\varepsilon)
$$

provided that the zero-range condition be maintained. In effect, the conditions $D_{\text {imp }} \gg \mathcal{R}, \lambda_{F}$ amount to neglecting multiple-interference scattering events. As in the homogeneous problem ${ }^{18}$ the dilute limit yields the following local self-energy for a random distribution of short-range impurity potentials:

$$
\left\langle\mathbf{r}|\Sigma(\varepsilon)| \mathbf{r}^{\prime}\right\rangle=n_{\text {imp }}(z) t(z) \delta\left(\mathbf{r}-\mathbf{r}^{\prime}\right),
$$

where the one-site $T$ matrix $t(z)$ is explicitly evaluated in Appendix A. It should be emphasized that this amounts to considering a subset of all possible diagrams, namely, those that are presumably dominant in the dilute limit.

This can be confirmed with an analysis in momentum space; the usual diagrammatic analysis leads in the dilute limit to an additive contribution ${ }^{18,10,13,14}$ from every locally homogeneous region

$$
\Sigma_{\mathbf{k}, \mathbf{k}}(\varepsilon ; z)=n_{\text {imp }}(z) t_{\mathbf{k}, \mathbf{k}^{\prime}}(\varepsilon ; z)
$$

to the total self-energy

$$
\left\langle\mathbf{k}|\Sigma(\varepsilon)| \mathbf{k}^{\prime}\right\rangle=\int \frac{d^{3} r}{V} \Sigma_{\mathbf{k}, \mathbf{k}^{\prime}}(\varepsilon ; z) e^{-i\left(\mathbf{k}-\mathbf{k}^{\prime}\right) \cdot \mathbf{r}},
$$

provided that $\mathcal{R} \ll a_{j}$; in effect, when $\mathcal{R}$ is small enough, the finiteness of the locally homogeneous region cannot be probed by the significantly more localized potential. Integration on a plane parallel to the layers, namely, $\int d^{2} \rho e^{-i\left(\mathbf{k}_{\|}-\mathbf{k}_{\|}^{\prime}\right) \cdot \boldsymbol{\rho}}=A \delta_{\mathbf{k}_{\|}, \mathbf{k}_{\|}^{\prime}}$, restores translational invariance in the "parallel" directions, because the reciprocal-space matrix elements $t_{\mathbf{k}, \mathbf{k}^{\prime}}\left(\varepsilon ; \mathbf{r}_{a}\right)=V\left\langle\mathbf{k}\left|t\left(\varepsilon ; \mathbf{r}_{a}\right)\right| \mathbf{k}^{\prime}\right\rangle=t_{a}$ are momentum independent (see Appendix A).

An alternative derivation and straightforward derivation of the result Eq. (3.12) is provided in Appendix B, where a particular solution of the integral equation (3.9) is considered, a procedure that amounts to the same choice of a subset of all self-energy diagrams.

\section{ONE-PARTICLE PROPAGATOR}

Let us now consider the "Green's function problem," i.e., the problem of finding the corresponding one-particle propagator. First, to simplify the form of the equations and to clarify the physics, I use the local mean free path $l_{\alpha}(z)$ that was defined in Eq. (2.1). In the language developed in the preceding section, this amounts to looking at the exponential decay of the one-particle propagator $\left\langle\mathbf{r}\left|\left\langle G_{\alpha}(\varepsilon)\right\rangle_{\mathcal{I}}\right| \mathbf{r}^{\prime}\right\rangle$ in the neighborhood of a given point $\mathbf{r}=(\boldsymbol{\rho}, z)$, where the individual spin channels $\alpha=\uparrow, \downarrow$ are treated independently, $\varepsilon=\varepsilon_{F}=\hbar^{2} k_{F}^{2} / 2 m$, and the real part of the self-energy can be absorbed as a redefinition of the energy reference level. Furthermore, as a result of the restoration of translational invariance in the plane of the layers (due to impurity averaging), it is most con- 
venient to work in the mixed $\left(\mathbf{k}_{\|}, z\right)$ representation, for which, according to the results of Sec. III,

$$
\left\langle\mathbf{k}_{\|}, z\left|\Sigma_{\alpha}(\varepsilon)\right| \mathbf{k}_{\|}^{\prime}, z^{\prime}\right\rangle=\delta_{\mathbf{k}_{\|}, \mathbf{k}_{\|}^{\prime}} \delta\left(z-z^{\prime}\right) \Sigma_{\alpha}(z),
$$

with a corresponding one-particle propagator

$$
\left\langle\mathbf{k}_{\|}, z\left|\left\langle G_{\alpha}(\varepsilon)\right\rangle_{\mathcal{I}}\right| \mathbf{k}_{\|}^{\prime}, z^{\prime}\right\rangle=\frac{2 m}{\hbar^{2}} \delta_{\mathbf{k}_{\|}, \mathbf{k}_{\|}^{\prime}} \mathcal{G}_{\alpha}\left(\mathbf{k}_{\|} ; z, z^{\prime}\right)
$$

even though $\mathcal{G}_{\alpha}\left(\mathbf{k}_{\|} ; z, z^{\prime}\right)$ is $\mathbf{k}_{\|}$dependent, I will omit this explicit dependence in the remainder of this section, as the main focus will be on its $z$ dependence.

Therefore the Green's function problem can be formulated by recalling that the one-particle propagator satisfies Dyson's equation (3.10), which I now rewrite as

$$
\left[\varepsilon-H_{0}-\Sigma_{\alpha}(\varepsilon)\right]\langle G(\varepsilon)\rangle_{\mathcal{I}}=\mathbb{1}
$$

where $\mathbb{1}$ is the unit one-particle operator. In the mixed $\left(k_{\|}, z\right)$ representation,

$$
\left\langle\mathbf{k}_{\|}, z\left|H_{0}\right| \mathbf{k}_{\|}^{\prime}, z^{\prime}\right\rangle=-\frac{\hbar^{2}}{2 m} \delta_{\mathbf{k}_{\|}, \mathbf{k}_{\|}^{\prime}}\left[\frac{d^{2}}{d z^{2}}-k_{\|}^{2}\right] \delta\left(z-z^{\prime}\right) .
$$

Then, substitution of Eqs. (4.1), (4.2), and (4.4) in Eq. (4.3) leads to the one-dimensional differential equation $^{8,24}$ for the reduced Green's function $\mathcal{G}_{\alpha}\left(z, z^{\prime}\right)$ in each spin channel $\alpha$,

$$
\left[\frac{d^{2}}{d z^{2}}+k_{\alpha}^{2}(z)\right] \mathcal{G}_{\alpha}\left(z, z^{\prime}\right)=\delta\left(z-z^{\prime}\right)
$$

where $k_{\alpha}(z)$ is a complex wave number or propagation constant given by the solution to

$$
k_{\alpha}^{2}(z)=\bar{k}^{2}+i \frac{k_{F}}{l_{\alpha}(z)}
$$

and $\bar{k}$ is the effective longitudinal momentum for electrons at the Fermi level, defined by

$$
\bar{k}=\sqrt{k_{F}^{2}-k_{\|}^{2}} \text {. }
$$

Notice that, in the quasiclassical limit,

$$
k_{\alpha}(z) \approx \bar{k}+i \frac{k_{F}}{2 \bar{k} l_{\alpha}(z)} .
$$

Equation (4.5) is the main result of this section. It is the final outcome of a diagrammatic analysis combined with a properly executed impurity-averaging procedure. Mathematically, it defines a typical Green's function problem for a Schrödinger-like equation with a complex potential. Most importantly, its physical content is exactly the one that was anticipated in Sec. II.

Of course, finding the solution to the differential equation (4.5) requires the knowledge of the boundary conditions satisfied at the end faces of the multilayer: $\partial_{<}$ (boundary at $z=0$ ) and $\partial_{>}$(boundary at $z=L$ ). For a finite system confined to a region of size $L$ by an infi- nite potential wall, Dirichlet boundary conditions are required to account for external size effects, that is, those due to the finiteness of the system: quantum size effects, when $k_{F} L \lesssim 1$, and quasiclassical external size effects, when $k_{F} L \gg 1$ but $l_{j, \alpha} \gtrsim L$. However, for the systems I discuss in this paper, $L \gg l_{j, \alpha} \gg \lambda_{F}$ (for all $j$ ), and size effects become asymptotically independent of any external boundary conditions, except for the detailed behavior of internal fields near the boundaries. Therefore, for the sake of simplicity, I replace Dirichlet boundary conditions by outgoing boundary conditions, as if the system were effectively infinite. Notice that the boundary conditions are taken to be outgoing for the retarded Green's function $G_{\alpha}^{\text {ret }}(\varepsilon)$. The Green's function calculated with these boundary conditions describes local transport behavior everywhere except near external boundaries, and global transport behavior or size effects.

The Green's function can be found as follows. Let $\psi_{<, \alpha}$ and $\psi_{>, \alpha}$ be the particular solutions to the homogeneous counterpart of Eq. (4.5), that is,

$$
\left[\frac{d^{2}}{d z^{2}}+k_{\alpha}^{2}(z)\right] \psi(z)=0
$$

that satisfy the proper boundary conditions at $\partial_{<}$and $\partial_{>}$, respectively. Then, the Green's function $\mathcal{G}_{\alpha}\left(z, z^{\prime}\right)$ is simply given by

$$
\mathcal{G}_{\alpha}\left(z, z^{\prime}\right)=\frac{1}{W\left[\psi_{<, \alpha}, \psi_{>, \alpha}\right]} \psi_{<, \alpha}\left(z_{<}\right) \psi_{>, \alpha}\left(z_{>}\right)
$$

where $W\left[\psi_{<, \alpha}, \psi_{>, \alpha}\right]$ is the Wronskian of $\psi_{<, \alpha}$ and $\psi_{>, \alpha}$. These auxiliary functions are given in every local region, for outgoing external boundary conditions and up to proportionality constants, by $\psi_{>, \alpha} \propto \exp \left[i k_{\alpha}(z) z\right]$ and $\psi_{<, \alpha} \propto \exp \left[-i k_{\alpha}(z) z\right]$. Of course, their complete expressions include factors that guarantee their continuity across internal boundaries.

I now introduce some additional useful notation. An arbitrary $N$-layered system consists of layers $\mathcal{L}_{j}$, with $z \in\left[z_{j-1}, z_{j}\right]$ and $j=1, \ldots, N$. For layer $\mathcal{L}_{j}$, let $k_{j, \alpha}$ be the local propagation constants (let $a_{j}$ and $l_{j, \alpha}$ be the layer thicknesses and mean free paths, respectively). The resolution $z=a_{j} u_{j}+z_{j-1}$, for $z$ in $\mathcal{L}_{j}$, is unique and permits the identification $z \equiv\left(j, u_{j}\right)$, which leads to a "layer-index notation," according to which the continuous variable $z$ can be replaced by both a discrete layer index $j$ and a reduced continuous dimensionless variable $u_{j} \in[0,1]$. For example, $\mathcal{G}_{\alpha}\left(z, z^{\prime}\right)=\mathcal{G}_{\alpha}^{j, j^{\prime}}\left(u_{j}, u_{j^{\prime}}\right)$, for $z \in \mathcal{L}_{j}$ and $z^{\prime} \in \mathcal{L}_{j^{\prime}}$, yielding an $N \times N$ matrix Green's function.

An additional simplification, which follows from the piecewise-linear character of multilayers, is that Eq. (4.9) becomes a Helmholtz equation in each layer, with solutions $\psi_{<, j, \alpha}$ and $\psi_{>, j, \alpha}$ that have to be determined by the external boundary conditions (at $\partial_{<}$or $\partial_{>}$) and by the internal boundary conditions (continuity of $\psi_{\alpha}$ and $\left.d \psi_{\alpha} / d z\right)$. Notice that this allows a simple treatment of the interfaces as regions of interdiffusion; instead, if disorder at or near the interfaces were interpreted in terms of interface roughness, this could be modeled in terms of 
$\delta$-function potentials at the interface, a model that reduces to thin layers of infinitesimal thickness (order of magnitude: $1 \AA$ ). Moreover, one is able in many cases to regard these regions as small enough to be modeled by $\delta$ functions, even when the physical mechanism is interdiffusion; see the interpretation of the resulting quasiclassical Fuchs-Sondheimer coefficients in the discussion following Eqs. (4.12)-(4.14). In the quasiclassical regime size effects can be completely characterized in terms of the set of dimensionless size parameters $\Lambda_{j, \alpha}=a_{j} / l_{j, \alpha}$ alone.

The Green's function for outgoing boundary conditions is, then,

$$
\begin{aligned}
\mathcal{G}_{\alpha}^{j, j^{\prime}}\left(u_{j}, u_{j^{\prime}}\right)= & (2 i \bar{k})^{-1} \exp \left[i \left(k_{j_{>}, \alpha} a_{j_{>}} u_{j_{>}}\right.\right. \\
& -k_{j_{<}, \alpha} a_{j_{<}} u_{j_{<}} \\
& \left.\left.+\sum_{j=j_{<}}^{j_{>}-1} k_{j, \alpha} a_{j}\right)\right]
\end{aligned}
$$

which in the quasiclassical limit becomes [cf. Eq. (4.8)]

$$
\begin{aligned}
\mathcal{G}_{\alpha}^{j, j^{\prime}}\left(u_{j}, u_{j^{\prime}}\right) \approx & (2 i \bar{k})^{-1} \exp \left[i \bar{k}\left|z-z^{\prime}\right|\right] \\
& \times \exp \left[-\frac{t}{2}\left(\Lambda_{j_{>}, \alpha} u_{j_{>}}-\Lambda_{j_{<}, \alpha} u_{j_{<}}\right.\right. \\
& \left.\left.+\Lambda_{\alpha}^{j_{<}, j_{>}-1}\right)\right] .
\end{aligned}
$$

In Eq. (4.12), the convention $\Lambda_{\alpha}^{j, j-1}=0$ was adopted, the terms

$$
\Lambda_{\alpha}^{j_{<}, j_{>}-1}=\frac{1}{t} \operatorname{Im}\left[\sum_{j=j_{<}}^{j_{>}-1} k_{j, \alpha} a_{j}\right] \approx \sum_{j=j_{<}}^{j_{>}-1} \Lambda_{j, \alpha}
$$

guarantee that the internal boundary conditions will be satisfied, $j_{<}\left(j_{>}\right)$denotes the layer corresponding to the smaller (larger) of $z$ and $z^{\prime}$, and the dimensionless variable

$$
t=\frac{k_{F}}{\bar{k}}
$$

was defined, with the following simple interpretation in the quasiclassical limit: For electrons at the Fermi level, the variable $\bar{k}$ can be interpreted as an effective longitudinal momentum [Eq. (4.7)]; then, for a quasiclassical electron propagating at an angle $\theta$ with respect to the $z$ axis, $t=(\cos \theta)^{-1}$.

In Eq. (4.12) the quasiclassical limit $k_{F} l_{j, \alpha} \gg 1$, for all $j$, has been used in order to arrive at what might be called a quasiclassical propagator, that is, one for which the "layer-diagonal" elements are $\mathcal{G}_{\alpha}\left(z, z^{\prime}\right) \propto e^{i \bar{k}\left|z-z^{\prime}\right|} e^{-\left(k_{F}\left|z-z^{\prime}\right| / 2 \bar{k} l_{j, \alpha}\right)}$, for $z, z^{\prime} \in \mathcal{L}_{j}$. Thus it follows that when an electron moves through a perpendicular distance $R_{\perp}$ in a given region, its coherent amplitude (one-particle propagator) decreases according to the exponential damping $\exp \left[-R_{\perp}\left(2 \cos \theta l_{j, \alpha}\right)^{-1}\right]$, and its "coherent intensity" as the square of that expression.
This characteristic exponential decay of the oneparticle propagator applies to any distance or region through which the electrons propagate; in particular this should be true of the additional interdiffusion layers. Thus, by treating interfaces as additional thin layers $\mathcal{S}_{j_{s}}$, with layer thicknesses $a_{j_{s}}^{(s)}$, and with characteristic surface-local mean free paths $l_{j_{s}, \alpha}^{(s)}$, a unified treatment of bulk and surface scattering, based upon Eq. (3.1), arises. Consequently, all the formulas derived so far apply also to a multilayered structure with interface scattering consisting of any number of layers $\mathcal{L}_{j_{b}}$ and interfaces $\mathcal{S}_{j_{s}}$. One could go one step further and replace the real interface regions by mathematical interfaces, assuming that $a_{j_{s}}^{(s)} \ll a_{j_{b}}^{(b)}$, and interpreting the exponential decay in the one-particle propagator in terms of an effective "coherent transmission coefficient" $T_{j_{s}, \alpha}^{(s)}(t)$, given by

$$
T_{j_{s}, \alpha}^{(s)}(t)=e^{-t \Lambda_{j_{s}, \alpha}^{(s)}},
$$

as dictated by the geometrical optics transmission picture. These transmission coefficients represent the fraction of electrons transmitted across the interface. The complementary fractions $1-T_{j_{s}, \alpha}^{(s)}(t)$ represent scattering at the interface, which is usually described as "diffusive." The coefficients $T_{j_{s}, \alpha}^{(s)}(t)$ play the same role as the ones required in a quasiclassical theory of multilayers with diffusive interfaces in order to match boundary conditions at the level of the distribution function, as first introduced by Carcia and Suna ${ }^{11}$ as a generalization of the specularity parameters of the Fuchs-Sondheimer theory. ${ }^{12}$ However, these new transmission coefficients, unlike the ones used by Carcia and Suna and in all subsequent quasiclassical papers, ${ }^{4}$ are not constant parameters but depend upon the angle of incidence $\theta$ of the electrons on the interface. In particular, these angle-dependent transmission coefficients favor the passage of electrons with nearly normal incidence and suppress exponentially the contribution from electrons moving nearly parallel to the interfaces.

This and other features of quasiclassical real-space transport can be more thoroughly understood by looking at an alternative way of deriving Eq. (4.12). In effect, Eq. (4.5) can be solved globally within a WKB approximation, that is, for small changes $\left|\Delta k_{\alpha}\right|$ in $k_{\alpha}(z)$ : $\left|\Delta k_{\alpha}\right| \ll k_{F}$, which amounts to the quasiclassical limit $1 / l_{\alpha}(z) \ll k_{F} ;$ then, ${ }^{25}$

$$
\begin{aligned}
\mathcal{G}_{\alpha}\left(z, z^{\prime}\right) & =\frac{1}{2 i \bar{k}} \exp \left[i \int_{z_{<}}^{z_{>}} d z k_{\alpha}(z)\right] \\
& \approx \frac{1}{2 i \bar{k}} \exp \left[i \bar{k}\left|z-z^{\prime}\right|-\frac{t}{2}\left|z-z^{\prime}\right| \xi_{\alpha}\left(z, z^{\prime}\right)\right],
\end{aligned}
$$

where

$$
\xi_{\alpha}\left(z, z^{\prime}\right)=\frac{1}{\left|z-z^{\prime}\right|} \int_{z_{<}}^{z_{>}} \frac{d z^{\prime \prime}}{l_{\alpha}\left(z^{\prime \prime}\right)} \propto\left\langle\Delta_{\alpha}\left(z^{\prime \prime}\right)\right\rangle_{z^{\prime \prime} \in\left[z_{<}, z_{>}\right]},
$$

with $z_{<}\left(z_{>}\right)$being the smaller (larger) of $z$ and $z^{\prime}$. 
Equation (4.18) defines a symmetric two-point function $\xi_{\alpha}\left(z, z^{\prime}\right)$ that gives the decay or attenuation constant in the one-particle propagator in terms of the average scattering between the two given points. Equations (4.17) and (4.18) are the equivalent of Eqs. (4.12) and (4.13), because

$$
\int_{z_{<}}^{z_{>}} \frac{d z^{\prime \prime}}{l_{\alpha}\left(z^{\prime \prime}\right)}=\left(\Lambda_{j_{>}, \alpha} u_{j_{>}}-\Lambda_{j_{<}, \alpha} u_{j_{<}}+\Lambda_{\alpha}^{j_{<}, j_{>}-1}\right) .
$$

This gives a simple interpretation of the expression (4.12) in terms of the effective average attenuation constant between the points $z_{<}$and $z_{>}$.

\section{REAL-SPACE KUBO APPROACH}

The linear electrical response of a system can be characterized in terms of conductivity functions. In this paper, I only consider time-independent phenomena. Then, all possible dc conductivity functions can be derived from the two-point conductivity tensor $\sigma\left(\mathbf{r}, \mathbf{r}^{\prime}\right)$, which establishes, in real space, the most general linear relationship between the current density $\mathbf{j}(\mathbf{r})$ at a given point $\mathbf{r}$ and the electric field $\mathbf{E}\left(\mathbf{r}^{\prime}\right)$ at a different point $\mathbf{r}^{\prime}$, namely,

$$
\mathbf{j}(\mathbf{r})=\int d^{3} r^{\prime} \boldsymbol{\sigma}\left(\mathbf{r}, \mathbf{r}^{\prime}\right) \cdot \mathbf{E}\left(\mathbf{r}^{\prime}\right) \text {. }
$$

The electric field in the formula above is the internal effective field in the medium, which usually differs from the external or applied field. It corresponds to what might be called "internal" conductivity, which can be directly calculated with the real-space Kubo formula ${ }^{13,17}$

$$
\begin{aligned}
\boldsymbol{\sigma}\left(\mathbf{r}, \mathbf{r}^{\prime}\right)= & \frac{4}{\pi} \frac{e^{2}}{\hbar}\left(\frac{\hbar^{2}}{2 m}\right)^{2} \\
& \times \sum_{\alpha, \beta=\uparrow, \downarrow} A_{\alpha \beta}\left(\mathbf{r}, \mathbf{r}^{\prime}\right) \stackrel{\leftrightarrow}{\nabla}_{\mathbf{r}} \stackrel{\leftrightarrow}{\nabla}_{\mathbf{r}^{\prime}} A_{\beta \alpha}\left(\mathbf{r}^{\prime}, \mathbf{r}\right)
\end{aligned}
$$

where $\stackrel{\leftrightarrow}{\nabla}_{\mathbf{r}}=\left(\vec{\nabla}_{\mathbf{r}}-\overleftarrow{\nabla}_{\mathbf{r}}\right)$, the greek indices label the two spin channels $(\alpha=\uparrow$ and $\downarrow)$, and

$$
\begin{aligned}
A_{\alpha \beta}\left(\mathbf{r}, \mathbf{r}^{\prime}\right) & =\frac{i}{2}\left[G_{\alpha \beta}^{\mathrm{ret}}\left(\mathbf{r}, \mathbf{r}^{\prime}\right)-G_{\alpha \beta}^{\mathrm{adv}}\left(\mathbf{r}, \mathbf{r}^{\prime}\right)\right] \\
& =-\operatorname{Im}\left[G_{\alpha \beta}^{\mathrm{ret}}\left(\mathbf{r}, \mathbf{r}^{\prime}\right)\right] .
\end{aligned}
$$

In this paper I will only analyze the particular but important case of collinear magnetization configurations, for which the Green's functions in Eqs. (5.2) and (5.3) become diagonal: $A_{\alpha \beta}\left(\mathbf{r}, \mathbf{r}^{\prime}\right)=\delta_{\alpha \beta} A_{\alpha}\left(\mathbf{r}, \mathbf{r}^{\prime}\right)$, for a choice of the quantization axis along the collinear direction, provided that spin-flip processes are neglected (two independent current model). Also, I would like to simplify the two-point formalism by using the in-plane symmetry of multilayers; due to in-plane translational invariance (homogeneity in the plane of the layers), the two-point conductivity satisfies the property $\boldsymbol{\sigma}\left(\mathbf{r}, \mathbf{r}^{\prime}\right)=\boldsymbol{\sigma}\left(\boldsymbol{\rho}-\boldsymbol{\rho}^{\prime} ; z, z^{\prime}\right)$, whence, a Fourier transform with respect to $\left(\boldsymbol{\rho}-\boldsymbol{\rho}^{\prime}\right)$ yields the conductivity function $\boldsymbol{\sigma}\left(\mathbf{k}_{\|} ; z, z^{\prime}\right)$; moreover, for uniform external fields, it is sufficient to consider the reduced two-point function

$$
\boldsymbol{\sigma}\left(z, z^{\prime}\right)=\boldsymbol{\sigma}\left(\mathbf{k}_{\|}=\mathbf{0} ; z, z^{\prime}\right)=\int d^{2} \rho \boldsymbol{\sigma}\left(\boldsymbol{\rho} ; z, z^{\prime}\right) .
$$

Then, resolving it into spin components,

$$
\boldsymbol{\sigma}\left(z, z^{\prime}\right)=\sum_{\alpha=\uparrow, \downarrow} \boldsymbol{\sigma}_{\alpha}\left(z, z^{\prime}\right)
$$

Eq. (5.2) reduces to

$$
\begin{aligned}
\boldsymbol{\sigma}_{\alpha}\left(z, z^{\prime}\right)= & \frac{4}{\pi} \frac{e^{2}}{\hbar} \int \frac{d^{2} k_{\|}}{(2 \pi)^{2}}\left[\frac{1}{2} k_{\|}^{2} \mathbb{1}_{\|}+\bar{k}^{2} \mathbf{e}_{z} \mathbf{e}_{z}\right] \\
& \times\left[\mathcal{A}_{\alpha}\left(\mathbf{k}_{\|} ; z, z^{\prime}\right)\right]^{2},
\end{aligned}
$$

where $\mathcal{A}_{\alpha}\left(\mathbf{k}_{\|} ; z, z^{\prime}\right)$ is the corresponding Fourier transform of Eq. (5.3), with the rescaling of Eq. (4.2), and associated with the retarded Green's function $\mathcal{G}_{\alpha}\left(\mathbf{k}_{\|} ; z, z^{\prime}\right)$ that was calculated in Sec. IV. In Eq. (5.6), the symmetry property of the Green's function $\mathcal{G}_{\alpha}\left(\mathbf{k}_{\|} ; z, z^{\prime}\right)=$ $\mathcal{G}_{\alpha}\left(\mathbf{k}_{\|} ; z^{\prime}, z\right)$ has been applied, as well as the operational identity $d / d z \approx i \bar{k}$ in the quasiclassical limit. From Eq. (4.17),

$$
\mathcal{A}_{\alpha}\left(\mathbf{k}_{\|} ; z^{\prime}, z\right)=\frac{1}{2 \bar{k}} \cos \left(\bar{k}\left|z-z^{\prime}\right|\right) \exp \left[-\frac{t}{2} \phi_{\alpha}\left(z, z^{\prime}\right)\right]
$$

where I have defined for convenience

$$
\phi_{\alpha}\left(z, z^{\prime}\right)=\left|z-z^{\prime}\right| \xi_{\alpha}\left(z, z^{\prime}\right)=\int_{z_{<}}^{z_{>}} \frac{d z^{\prime \prime}}{l_{\alpha}\left(z^{\prime \prime}\right)}
$$

[cf. Eq. (4.18)]. Notice that the real-space Green's function $\mathcal{G}_{\alpha}$ oscillates rapidly with a characteristic spatial frequency of the order of $k_{F}$, a property which is relevant for quantum size effects; in the quasiclassical limit, the linear response can be probed only over distances of at least a few times $\lambda_{F}$, and Eq. (5.6) is automatically averaged. The momentum integral in Eq. (5.6) is reduced to exponential integral functions ${ }^{26}$

$$
E_{n}(x)=\int_{1}^{\infty} \frac{d t}{t^{n}} e^{-t x}
$$

in effect, calling $C_{D}=n e^{2} / 2 m v_{F}=e^{2} k_{F}^{2} / 6 \pi^{2} \hbar$, from Eqs. (5.6) and (5.7), the two-point conductivity functions are given by

$$
\sigma_{\alpha}^{(\|)}\left(z, z^{\prime}\right)=\frac{3 C_{D}}{4}\left\{E_{1}\left[\phi_{\alpha}\left(z, z^{\prime}\right)\right]-E_{3}\left[\phi_{\alpha}\left(z, z^{\prime}\right)\right]\right\}
$$

and

$$
\sigma_{\alpha}^{(\perp)}\left(z, z^{\prime}\right)=\frac{3 C_{D}}{2} E_{3}\left[\phi_{\alpha}\left(z, z^{\prime}\right)\right]
$$

Equations (5.10) and (5.11) introduce a nonlocal linear transport theory of multilayered structures. The characteristic length for nonlocality is precisely the mean free 
path. This nonlocality, called spatial dispersion, is an essential ingredient in any inhomogeneous system. This is precisely the general framework that we set out to develop in this paper. It should be pointed out that such a general framework is absent in all treatments based on the Boltzmann equation, ${ }^{4}$ and it was not developed in the momentum space Kubo approach of Levy et al. ${ }^{7}$ which is restricted to one-point functions, thus requiring a "local approximation" for the calculation of CPP conductivities. ${ }^{19}$ The success in developing a complete nonlocal theory is entirely due to the choice of a "realspace" approach.

\section{GLOBAL PROPERTIES}

From the two-point conductivity derived in Sec. V, the measurable or global properties can be derived by imposing proper constraints on the currents or on the fields.

In order to gain insight into the physics of GMR, it is useful to define two limiting cases: ${ }^{27}$ (i) the local limit, when $l_{j, \alpha} \ll a_{j}$, for all $j$, and (ii) the homogeneous limit, when $l_{j, \alpha} \gg a_{j}$, for all $j$. In the local limit, as all mean free paths are negligibly small with respect to the inhomogeneity lengths, the linear response within a given layer is like that of an infinite medium made up of the same material and with the same type of distribution of impurities; as a result of the local nature of the response, the neighborhoods of all points in the medium are uncorrelated and become effectively independent resistors, which means they can be added as "classical" resistors: in series for current in the plane of the layers (CIP) and in parallel for current perpendicular to the planes of the layers. On the other hand, in the homogeneous limit, as all mean free paths are effectively very large, the linear response becomes very nonlocal and contributions to the resistivity from all layers are added; in effect, the electron propagates, probing all the scattering within a mean free path, which includes several layers, and it therefore averages all sorts of scattering in the medium. If the amount of local scattering in channel $\alpha$ is $\Delta_{\alpha}(z)$, then the global resistance in the homogeneous limit is given by the average $\left\langle\Delta_{\alpha}(z)\right\rangle$ (as the local resistivity is proportional to the local scattering rate); this process is called self-averaging, and effectively it amounts to adding all local resistors in series.

The following conclusions can be immediately drawn. First, for the CPP geometry both limiting cases can be described with a "series resistor model;" instead, for the CIP geometry very different limiting behaviors are exhibited: series resistor model in the homogeneous limit and parallel resistor model in the local limit. Thus size effects are much more manifest in the CIP case, where complete transport theories should provide the correct interpolation between the two limiting cases; typically, this interpolation is given by linear combinations of exponential functions. An interesting possibility is that the CPP conductances be just given by the series resistor model for all length scales; below we see that this is precisely the case. Second, the series resistor model yields maximum magnetoresistance because a short-circuit effect between the two channels actually takes place for resistors in series (within one channel). This is always true for the CPP case (see below) and for the homogeneous limit. Instead, when a parallel resistor model holds, all resistors (including those corresponding to the two different channels) are added in parallel; as a consequence, the final equivalent combination of resistors is the same for the antiferromagnetic and ferromagnetic configurations, and leads to zero magnetoresistance. Then, the local limit of the CIP geometry has no magnetoresistance, in sharp contrast with the CPP geometry. An important corollary is that the CPP magnetoresistance is always larger than the CIP magnetoresistance; as the local limit is approached their differences become rather dramatic.

The derivation of global properties is straightforward for the CIP geometry. ${ }^{7}$ As our results are quasiclassical, they are essentially in agreement with those derived from the Boltzmann equation; ${ }^{4-6,28}$ as this has been done in several papers I will not write here the rather cumbersome general expressions that follow from this theory. ${ }^{29}$ The only caveat is again that interface scattering should be modeled with interface layers, which physically represent the effect of interdiffusion. ${ }^{16,8}$

On the other hand, the problem for the CPP geometry requires further analysis. For the CPP geometry the current is uniform throughout, and even though a uniform electric field is applied to the layers, the actual field in the solid varies from one layer to another and is spin dependent. In the remainder of this section I will work out the consequences of this real-space model for the CPP geometry.

In order to focus on the spin-dependent nature of the scattering and on the implications of this for the internal fields, I explicitly resolve the total current into two spin currents, that is,

$$
j=\sum_{\alpha=\uparrow, \downarrow} j_{\alpha}(z)
$$

where the linear response for each individual spin current is

$$
j_{\alpha}(z)=\int d z^{\prime} \sigma_{\alpha}^{(\perp)}\left(z, z^{\prime}\right) E_{\alpha}\left(z^{\prime}\right)
$$

with spin-dependent conductivities $\sigma_{\alpha}^{(\perp)}\left(z, z^{\prime}\right)$ given by Eq. (5.11). In Eq. (6.2) the fields are explicitly spin dependent because they are subject to the simultaneous constraints

$$
\boldsymbol{\nabla} \cdot \mathbf{j}_{\alpha}(\mathbf{r})=0
$$

that is, the continuity equation for each spin channel in the limit of no spin mixing. In particular, $j_{\alpha}(z)$ is independent of $z$, i.e., from Eqs. (5.11), (6.2), and (6.3),

$$
j_{\alpha}=\frac{3 C_{D}}{2} \int d z^{\prime} \int_{1}^{\infty} \frac{d t}{t^{3}} \exp \left[-t \phi_{\alpha}\left(z, z^{\prime}\right)\right] E_{\alpha}\left(z^{\prime}\right)
$$

[with $\phi_{\alpha}\left(z, z^{\prime}\right)$ defined through Eqs. (4.18) and (5.8)] is a constant.

Therefore finding the global CPP resistivity leads to 
solving the integral equation (6.4). This can be interpreted as searching for the steady-state internal field distribution that is consistent with current conservation. This turns out to be a trivial problem; in effect, the form of the exponent, Eq. (4.18), and the piecewise-constant nature of the scattering imply for an infinite multilayered structure $\left(L \gg l_{j, \alpha}, a_{j}\right)$ that

$$
\begin{aligned}
t \int \frac{d z^{\prime}}{l_{\alpha}\left(z^{\prime}\right)} & \exp \left[-t \int_{z_{<}}^{z_{>}} \frac{d z^{\prime \prime}}{l_{\alpha}\left(z^{\prime \prime}\right)}\right] \\
& =2 \int_{0}^{1} d\left(\exp \left[-t \int_{z_{<}}^{z_{>}} \frac{d z^{\prime \prime}}{l_{\alpha}\left(z^{\prime \prime}\right)}\right]\right)=2
\end{aligned}
$$

(two perfect differentials: one for $z>z^{\prime}$ and one for $\left.z<z^{\prime}\right)$. This gives the solution for the internal field by direct inspection, ${ }^{25}$ if one inverts the order of integration in Eq. (6.4), that is,

$$
E_{\alpha}(z)=\frac{j_{\alpha}}{C_{D}} \frac{1}{l_{\alpha}(z)}
$$

which amounts to an internal field that is inversely proportional to the local mean free path. An alternative but lengthier proof follows by using the discrete version of the two-point conductivity, i.e., integrating Eq. (6.4) layer by layer; after an infinite series of cancellations (provided that $\left.L \gg l_{j, \alpha}, a_{j}\right)$, one obtains the same result as before.

With the result that $E_{\alpha}(z) \propto 1 / l_{\alpha}(z)$, it follows that the contribution to the CPP resistivity due to channel $\alpha$ is

$$
\rho_{\mathrm{CPP}, \alpha}=\frac{\langle E\rangle}{j_{\alpha}}=\left(C_{D}\right)^{-1}\left\langle\frac{1}{l_{\alpha}(z)}\right\rangle \propto\left\langle\Delta_{\alpha}(z)\right\rangle,
$$

which is solely determined by the average scattering in the medium. Therefore the CPP resistivity is selfaveraging and given by the series resistor model, not just for the local and self-averaging limiting cases, but for all length scales. Then, from the two-current model,

$$
\sigma_{\mathrm{CPP}}=C_{D} \sum_{\alpha=\uparrow, \downarrow}\left\langle\left(\frac{1}{l_{\alpha}(z)}\right\rangle\right)^{-1}
$$

This is the result that had been predicted in Ref. 19 and that has been found to be in impressive agreement with experimental results. ${ }^{3}$

\section{CONCLUSIONS}

In this paper, I have analyzed the electrical transport properties of magnetic multilayers. Although most results rely on the metallic character of these structures, I placed particular emphasis on the magnetoconductance due to a reorientation of the magnetization vectors of the different regions of these inhomogeneous structures. This is motivated by the huge interest attracted by the phenomenon called giant magnetoresistance, whose un- derlying physical origin is believed to be spin-dependent scattering.

The main purpose of this paper has been to develop a general solution of the Kubo formula for metallic multilayers. In particular, I have clarified the emergence of the quasiclassical regime for multilayers from the quantumstatistical Kubo formula, and indicated where such a regime is to be found experimentally in terms of the adjustable parameters of the model.

The solution I developed from the Kubo formula has the following features. First, it is a real-space approach, i.e., it is based upon the real-space Kubo formula. Second, the Green's function problem is tackled only after the self-energy has been cast into a simple form in the dilute limit. Third, the Green's function problem is reduced in real space to the solution of a differential equation with matching boundary conditions at interfaces. The transport coefficients that are actually computed in this real-space approach amount to the two-point conductivity function, which expresses the most general linear and nonlocal relationship between the current density and the internal fields in the solid.

Using this real-space Kubo approach, I derived general analytical formulas for the two-point conductivity for the quasiclassical regime. One interesting feature of this clarification is that it suggests an improved form for the phenomenological coherent transmission coefficients of the quasiclassical approach, an improvement that includes both an angle dependence and a clear physical interpretation. The striking differences between the CIP and CPP conductances and magnetoconductances were discussed, and the emergence of simple limiting cases was recovered from this more general formalism. In particular, I derived a proof of the so-called series resistor model for CPP.

Finally, I would like to emphasize that this real-space theory constitutes a first approach towards a more comprehensive theory of transport in metallic multilayers. Generalizations of the formalism that are immediately suggested are transport in the presence of noncollinear magnetization configurations for multilayers, transport in three-dimensionally inhomogeneous magnetic structures such as magnetic granular solids, ${ }^{30}$ and transport in multilayers in the presence of "superlattice potentials." 31 These generalizations involve new effects that can be naturally dealt with within the real-space approach, and will be presented elsewhere.

In conclusion, the general real-space Kubo approach developed in this paper provides a remarkably simple, both intuitive and analytic, understanding of magnetotransport in multilayers, and it constitutes a rather flexible tool that naturally paves the way for further generalizations.

\section{ACKNOWLEDGMENTS}

I would like to acknowledge useful discussions with Professor P. M. Levy and Dr. S. Zhang. This work was supported in part by the Office of Naval Research Grant No. N00014-91-J-1695. 


\section{APPENDIX A: PROPERTIES OF ZERO-RANGE POTENTIALS}

A zero-range potential of coupling strength $\lambda$, i.e.,

$$
v(\mathbf{r})=\lambda \delta\left(\mathbf{r}-\mathbf{r}_{0}\right)
$$

has real-space matrix elements

$$
\left\langle\mathbf{r}|v| \mathbf{r}^{\prime}\right\rangle=\lambda \delta\left(\mathbf{r}-\mathbf{r}_{0}\right) \delta\left(\mathbf{r}^{\prime}-\mathbf{r}_{0}\right)
$$

that is, it is "bilocal." The corresponding one-site $T$ matrix is given by the solution to Eq. (3.11), that is, using symbolic operator notation,

$$
t=v\left(1-G_{0} v\right)^{-1}=v+v G^{0} v+v G^{0} v G^{0} v+\cdots ;
$$

its real-space representation always has two Dirac $\delta$ functions in excess of integration variables, thus yielding the bilocal one-site $T$ matrix

$$
\left\langle\mathbf{r}|t| \mathbf{r}^{\prime}\right\rangle=\lambda(1-\lambda S)^{-1} \delta\left(\mathbf{r}-\mathbf{r}_{0}\right) \delta\left(\mathbf{r}^{\prime}-\mathbf{r}_{0}\right),
$$

where $S=g^{0}(0)$ for $g(R)=g\left(\left|\mathbf{r}-\mathbf{r}^{\prime}\right|\right)=G^{0}\left(\mathbf{r}, \mathbf{r}^{\prime}\right)$; notice that $S$ is proportional to the $\operatorname{trace} \operatorname{tr}\left[G_{0}(\varepsilon)\right]$ of the unperturbed one-particle propagator, which could be written in terms of the unperturbed density of states per unit volume, ${ }^{14} \rho_{0}(\varepsilon)$.

The following result then follows from Eqs. (3.12) and (A4): For a random distribution of zero-range potentials, if the self-energy happens to be local and given Eq. (3.12), i.e.,

$$
\left\langle\mathbf{r}|\Sigma| \mathbf{r}^{\prime}\right\rangle=\Sigma(\mathbf{r}) \delta\left(\mathbf{r}-\mathbf{r}^{\prime}\right)
$$

then its diagonal elements are

$$
\Sigma(\mathbf{r})=n(\mathbf{r}) \lambda(\mathbf{r})[1-\lambda(\mathbf{r}) S]^{-1},
$$

where $n(\mathbf{r})=n_{\text {imp }}(\mathbf{r})$ in this appendix. The corresponding impurity-averaged off-shell $T$ matrix, which is the solution to Eq. (3.9), namely,

$$
\langle T(\varepsilon)\rangle_{\mathcal{I}}=\Sigma(\varepsilon)\left[1-G^{0}(\varepsilon) \Sigma(\varepsilon)\right]^{-1}
$$

has the following symbolic form [from Eqs. (A5) and (A6)]:

$T\left(\mathbf{r}, \mathbf{r}^{\prime}\right)=n(\mathbf{r}) \lambda(\mathbf{r})\left[1-\lambda(\mathbf{r}) S-\lambda(\mathbf{r}) n(\mathbf{r}) G^{0}\right]^{-1}\left(\mathbf{r}, \mathbf{r}^{\prime}\right)$

where the notation $T\left(\mathbf{r}, \mathbf{r}^{\prime}\right)=\left\langle\mathbf{r}\left|\langle T(\varepsilon)\rangle_{\mathcal{I}}\right| \mathbf{r}^{\prime}\right\rangle$ was used.

\section{APPENDIX B: INTEGRAL EQUATION FOR THE TOTAL $T$ MATRIX}

In this appendix I give an alternative derivation of the result of Sec. III that in the dilute limit the self-energy is given by Eq. (3.12) for zero-range potentials.
The basic equation for the total $T$ matrix is given by Eq. (3.7), which can be iterated to give the perturbative series

$$
T=\mathcal{V}+\mathcal{V} G^{0} \mathcal{V}+\mathcal{V} G^{0} \mathcal{V} G^{0} \mathcal{V}+\cdots
$$

(where I have omitted explicit reference to the energy dependence).

For a zero-range potential $v_{a}(\mathbf{r})=\lambda_{a} \delta\left(\mathbf{r}-\mathbf{r}_{a}\right)$, the real-space matrix elements of the total $T$ matrix are

$$
\begin{aligned}
\left\langle\mathbf{r}|T| \mathbf{r}^{\prime}\right\rangle= & \sum_{a} \lambda_{a} \delta\left(\mathbf{r}-\mathbf{r}_{a}\right) \delta\left(\mathbf{r}^{\prime}-\mathbf{r}_{a}\right) \\
& +\sum_{a} \lambda_{a} \delta\left(\mathbf{r}-\mathbf{r}_{a}\right) \\
& \times \int d^{3} r_{2} G^{0}\left(\mathbf{r}_{a}, \mathbf{r}_{2}\right)\left\langle\mathbf{r}_{2}|T| \mathbf{r}^{\prime}\right\rangle .
\end{aligned}
$$

In this appendix, for the sake of notational simplicity, I do not explicitly write the spin-channel index $\alpha$ and I use the symbol $n(\mathbf{r})$ rather than $n_{\text {imp }}(\mathbf{r})$ for the concentration of impurities. Using a "multiple-site technique"

$$
T=\sum_{a} \tau_{a}
$$

one obtains

$$
\begin{aligned}
\sum_{a} \tau_{a}\left(\mathbf{r}, \mathbf{r}^{\prime}\right)= & \sum_{a} \lambda_{a} \delta\left(\mathbf{r}-\mathbf{r}_{a}\right) \delta\left(\mathbf{r}^{\prime}-\mathbf{r}_{a}\right) \\
& +\sum_{a, b} \lambda_{a} \delta\left(\mathbf{r}-\mathbf{r}_{a}\right) \\
& \times \int d^{3} r_{2} G^{0}\left(\mathbf{r}_{a}, \mathbf{r}_{2}\right) \tau_{b}\left(\mathbf{r}_{2}, \mathbf{r}^{\prime}\right)
\end{aligned}
$$

where $\tau_{a}\left(\mathbf{r}, \mathbf{r}^{\prime}\right)=\left\langle\mathbf{r}\left|\tau_{a}\right| \mathbf{r}^{\prime}\right\rangle$

Applying the impurity-averaging procedure described in Sec. III, and using the notation $T\left(\mathbf{r}, \mathbf{r}^{\prime}\right)=$ $\left\langle\mathbf{r}\left|\langle\boldsymbol{T}(\varepsilon)\rangle_{\mathcal{I}}\right| \mathbf{r}^{\prime}\right\rangle$, one finds that the real-space matrix elements of the total impurity-averaged $T$ matrix are

$$
T\left(\mathbf{r}, \mathbf{r}^{\prime}\right)=\int d^{3} r_{1} n\left(\mathbf{r}_{1}\right) \tau_{\mathbf{r}_{1}}\left(\mathbf{r}, \mathbf{r}^{\prime}\right)
$$

where $\tau_{\mathbf{r}_{a}}\left(\mathbf{r}, \mathbf{r}^{\prime}\right)=\tau_{a}\left(\mathbf{r}, \mathbf{r}^{\prime}\right)$, and the following integral equation is satisfied:

$$
\begin{aligned}
T\left(\mathbf{r}, \mathbf{r}^{\prime}\right)= & n(\mathbf{r}) \lambda(\mathbf{r}) \delta\left(\mathbf{r}-\mathbf{r}^{\prime}\right) \\
& +n(\mathbf{r}) \lambda(\mathbf{r}) \int d^{3} r_{2} G^{0}\left(\mathbf{r}, \mathbf{r}_{2}\right) \tau_{\mathbf{r}}\left(\mathbf{r}_{2}, \mathbf{r}^{\prime}\right) \\
& +n(\mathbf{r}) \lambda(\mathbf{r}) \int d^{3} r_{2} G^{0}\left(\mathbf{r}, \mathbf{r}_{2}\right) T\left(\mathbf{r}_{2}, \mathbf{r}^{\prime}\right) .
\end{aligned}
$$

In the dilute limit, the second term on the right-hand side can be replaced with the approximation

$$
n(\mathbf{r}) \int d^{3} r_{2} G^{0}\left(\mathbf{r}, \mathbf{r}_{2}\right) \tau_{\mathbf{r}}\left(\mathbf{r}_{2}, \mathbf{r}^{\prime}\right) \approx S T\left(\mathbf{r}, \mathbf{r}^{\prime}\right)
$$

where $S=g^{0}(0)=G^{0}(\mathbf{r}, \mathbf{r})$ was defined in Appendix A; this can be understood as having $\mathbf{r}_{2}$ and $\mathbf{r}^{\prime}$ close to $\mathbf{r}$ in the "dilute approximation." 
From Eqs. (B6) and (B7), using symbolic operator notation,

$$
T\left(\mathbf{r}, \mathbf{r}^{\prime}\right)=n(\mathbf{r}) \lambda(\mathbf{r})\left[1-\lambda(\mathbf{r}) S-\lambda(\mathbf{r}) n(\mathbf{r}) G^{0}\right]^{-1}\left(\mathbf{r}, \mathbf{r}^{\prime}\right),
$$

which is identical to Eq. (A8). Therefore, by comparison with the analysis of Appendix A, one concludes that the self-energy is local, with the real-space representation

$$
\begin{aligned}
\Sigma\left(\mathbf{r}, \mathbf{r}^{\prime}\right) & =\delta\left(\mathbf{r}-\mathbf{r}^{\prime}\right) n(\mathbf{r}) t(\mathbf{r}) \\
& =\delta\left(\mathbf{r}-\mathbf{r}^{\prime}\right) n(\mathbf{r}) \lambda(\mathbf{r})[1-\lambda(\mathbf{r}) S]^{-1},
\end{aligned}
$$

and the results of Sec. III follow.
${ }^{1}$ M. N. Baibich, J. M. Broto, A. Fert, F. Nguyen Van Dau, F. Petroff, P. Etienne, G. Creuzet, A. Friederich, and J. Chazelas, Phys. Rev. Lett. 61, 2472 (1988).

2 A. Barthélémy, A. Fert, M. N. Baibich, S. Hadjoudj, F. Petroff, P. Etienne, R. Cabanel, S. Lequien, and G. Creuzet, J. Appl. Phys. 67, 5908 (1990); G. Binach, P. Grunberg, F. Saurenbach, and W. Zinn, Phys. Rev. B 39 , 4828 (1989); J. J. Krebs, P. Lubitz, A. Chaiken, and G. A. Prinz, Phys. Rev. Lett. 63, 1645 (1989); S. S. P. Parkin, N. More, and K. P. Roche, ibid. 64, 2304 (1990); B. Dieny, V. S. Speriosu, S. S. P. Parkin, B. A. Gurney, D. R. Wilhoit, and D. Mauri, Phys. Rev. B 43, 1297 (1991); S. S. P. Parkin, R. Bhadra, and K. P. Roche, Phys. Rev. Lett. 67, 140 (1991); T. Takahata, S. Araki, and T. Shinjo, J. Magn. Magn. Mater. 82, 827 (1989); A. Chaiken, G. A. Prinz, and J. J. Krebs, J. Appl. Phys. 67, 4892 (1990); N. Hosoito et al., J. Phys. Soc. Jpn. 59, 1925 (1990); D. H. Mosca, F. Petroff, A. Fert, P. A. Schroeder, W. P. Pratt, Jr., and R. Loloee, J. Magn. Magn. Mater. 94, L1 (1991); F. Petroff et al., Phys. Rev. B 44, 5355 (1991); V. S. Speriosu et al., ibid. 44, 5358 (1991); S. S. P. Parkin, Z. G. Lin, and D. J. Smith, Appl. Phys. Lett. 58, 2710 (1991); B. Rodmacq et al., Phys. Rev. B 46, 1206 (1992); M. A. M. Gijs and M. Okada, ibid. 46, 2908 (1992); Y. Obi et al., J. Magn. Magn. Mater. 104-107, 1747 (1992); E. E. Fullerton et al., Phys. Rev. Lett. 68, 859 (1992); Y. Saito, S. Hashimoto, and K. Inomata, Appl. Phys. Lett. 60, 2436 (1992); J. Kohlhepp et al., J. Magn. Magn. Mater. 111, 231 (1992); P. Baumgart et al., J. Appl. Phys. 69, 4792 (1992).

${ }^{3}$ W. P. Pratt, Jr., S.-F. Lee, J. M. Slaughter, R. Loloee, P. A. Schroeder, and J. Bass, Phys. Rev. Lett. 66, 3060 (1991); S.-F. Lee, W. P. Pratt, Jr., R. Loloee, P. A. Schroeder, and J. Bass, Phys. Rev. B 46, 548 (1992); M. A. M. Gijs, S. K. J. Lenczowski, and J. B. Giesbers, Phys. Rev. Lett. 70, 3343 (1993).

${ }^{4}$ R. E. Camley and J. Barnás, Phys. Rev. Lett. 63, 664 (1989); J. Barnás, A. Fuss, R. E. Camley, P. Grunberg, and W. Zinn, Phys. Rev. B 42, 8110 (1990).

5 A. Barthélémy and A. Fert, Phys. Rev. B 43, 13124 (1991).

${ }^{6}$ B. Dieny, Europhys. Lett. 17, 261 (1992).

${ }^{7}$ P. M. Levy, S. Zhang, and A. Fert, Phys. Rev. Lett. 65, 1643 (1990); S. Zhang, P. M. Levy, and A. Fert, Phys. Rev. B 45, 8689 (1992).

${ }^{8}$ H. E. Camblong and P. M. Levy, Phys. Rev. Lett. 69, 2835 (1992); J. Magn. Magn. Mater. 121, 446 (1993).

${ }^{9}$ A. Fert and I. A. Campbell, Phys. Rev. Lett. 21, 1190 (1968); A. Fert, J. Phys. C 2, 1784 (1969); A. Fert and I. A. Campbell, J. Phys. F 6, 849 (1976); I. A. Campbell and A. Fert, in Ferromagnetic Materials, edited by E. P. Wohlfarth (North-Holland, Amsterdam, 1982), Vol. 3, p. 769.

${ }^{10}$ G. D. Mahan, Many-Particle Physics, 2nd ed. (Plenum, New York, 1990), Sec. 3.8 and Chap. 7.

${ }^{11}$ R. F. Carcia and A. Suna, J. Appl. Phys. 54, 2000 (1983).
${ }^{12}$ K. Fuchs, Proc. Philos. Cambridge Soc. 34, 100 (1938); E. H. Sondheimer, Adv. Phys. 1, 1 (1952).

${ }^{13}$ G. Rickayzen, Green's Functions and Condensed Matter (Academic Press, San Diego, 1980), Chap. 4.

${ }^{14}$ S. Doniach and E. H. Sondheimer, Green's Functions for Solid State Physicists (Benjamin, Reading, MA, 1974).

${ }^{15}$ Z. Tešanović, M. V. Jaric, and S. Maekawa, Phys. Rev. Lett. 57, 2760 (1986).

${ }^{16}$ B. L. Johnson and R. E. Camley, Phys. Rev. B 44, 9997 (1991).

${ }^{17}$ D. S. Fisher and P. A. Lee, Phys. Rev. B 23, 6851 (1981); J. Rammer, Rev. Mod. Phys. 63, 781 (1991).

${ }^{18}$ W. Kohn and J. M. Luttinger, Phys. Rev. 108, 590 (1957).

${ }^{19}$ S. Zhang and P. M. Levy, J. Appl. Phys. 69, 4786 (1991). This paper presents a prediction of self-averaging behavior based on a proof that is flawed because the full-fledged two-point structure of the linear response is not explored.

${ }^{20}$ N. Trivedi and N. W. Ashcroft, Phys. Rev. B 38, 12298 (1988); G. Fishman and D. Calecki, Phys. Rev. Lett. 62, 1302 (1989).

${ }^{21}$ J. S. Langer and T. Neal, Phys. Rev. Lett. 16, 984 (1966); B. L. Al'tshuler, A. G. Aronov, D. E. Khmelnitskii, and A. I. Larkin, in Quantum Theory of Solids, edited by I. M. Lifshits (Mir, Moscow, 1982); G. Bergmann, Phys. Rev. B 28, 2914 (1983); Phys. Rep. 107, 1 (1984); D. E. Khmel'nitskii, Physica B 126, 235 (1984); C. P. Enz, A Course on ManyBody Theory Applied to Solid-State Physics (World Scientific, Singapore, 1992), Chap. 3.

22 J. R. Asik, M. A. Ball, and C. P. Slichter, Phys. Rev. Lett. 16, 740 (1966); P. Monod and S. Schultz, J. Phys. (Paris) 43, 393 (1982).

${ }^{23}$ Y. Yafet, J. Appl. Phys. 39, 85 (1968); 42, 1564 (1971).

${ }^{24}$ Equation (4.5) has been derived within a different quantum Kubo approach in A. Vedyayev, B. Dieny, and N. Ryzhanova, Europhys. Lett. 19, 329 (1992).

${ }^{25}$ H. E. Camblong and P. M. Levy, J. Appl. Phys. 73, 5533 (1993).

${ }^{26}$ Handbook of Mathematical Functions, edited by $M$. Abramowitz and I. A. Stegun (Dover Publications, New York, 1965), Chap. 5.

${ }^{27}$ H. E. Camblong, S. Zhang, and P. M. Levy, Phys. Rev. B 47, 4735 (1993).

${ }^{28}$ R. Q. Hood and L. M. Falicov, Phys. Rev. B 46, 8287 (1992).

${ }^{29}$ H. E. Camblong, Ph.D. thesis, New York University, 1993.

${ }^{30}$ A. E. Berkowitz, J. R. Mitchell, M. J. Carey, A. P. Young, S. Zhang, F. E. Spada, F. T. Parker, A. Hutten, and G. Thomas, Phys. Rev. Lett. 68, 3745 (1992); J. Q. Xiao, J. S. Jiang, and C. L. Chien, ibid. 68, 3749 (1992); Phys. Rev. B 46, 9266 (1992); P. Xiong, G. Xiao, J. Q. Wang, J. Q. Xiao, S. Jiang, and C. L. Chien, Phys. Rev. Lett. 69, 3220 (1992).

${ }^{31}$ P. M. Levy, Z.-P. Shi, S. Zhang, H. E. Camblong, and J. L. Fry, J. Magn. Magn. Mater. 121, 357 (1993). 